\title{
Inhibition of Semaphorin 4D/Plexin-B1 signal inhibits the subchondral bone loss in
} early-stage osteoarthritis of temporomandibular joint

Zhaoyichun Zhang ${ }^{1,2 \#}$, Lei Lu ${ }^{2 \#}$, Tao Ye ${ }^{2 \#}$, Shibin $\mathrm{Yu}^{2}$, Jing Zhang ${ }^{2}$, Mian Zhang ${ }^{2}$, Feng $\mathrm{He}^{2}$, Qian Liu $^{2}$, Jianying Feng ${ }^{1 *}$, Hongxu Yang ${ }^{2 *}$

1. School of Stomatology, Zhejiang Chinese Medicine University, Hang Zhou, China, 310054.

2. State Key Laboratory of Military Stomatology \& National Clinical Research Center for Oral Diseases \& Shaanxi Key Laboratory of Stomatology, Department of Oral Anatomy and Physiology and TMD, School of Stomatology, The Fourth Military Medical University, Xi'an, China, 710032.

\section{* Corresponding Authors:}

Hongxu Yang: Department of Oral Anatomy and Physiology and TMD, School of Stomatology, The Fourth Military Medical University, 145 Changle West Road, Xi'an, China, 710032, yanghongxushmily@163.com.

Jianying Feng: School of Stomatology, Zhejiang Chinese Medicine University, 548 binwen Road, Hangzhou, China, 310054, twohorsejy@163.com.

\# Zhaoyichun Zhang, Lei Lu and Tao Ye contributed equally to the present study. 


\begin{abstract}
Subchondral bone loss is an important pathological feature of early-stage temporomandibular joint (TMJ) osteoarthritis (OA). Previous studies focused mainly on the bone resorption by osteoclasts in early-stage OA, but the bone formation feature has not drawn enough attention. Sema4D/Plexin-B1 is a pair of molecules expressed by osteoclast/osteoblast, which is capable of inhibiting bone formation by osteoblasts. The present study found that subchondral bone loss in early-stage TMJ OA was accompanied by up-regulated expression of Sema4D in cartilage and subchondral bone and Plexin-B1 in subchondral bone. Reducing Sema4D level could inhibit the subchondral bone loss and cartilage degeneration of early-stage TMJ OA. In vitro, results revealed that Sema4D could reduce the expression of osteocalcin (OCN) and alkaline phosphatase (ALP), and increase the migrating capability of Plexin-B1-positive osteoblasts. Our results revealed that elevated Sema4D expression in early-stage TMJ OA might decrease the bone formation activity of osteoblasts in the subchondral bone by binding to Plexin-B1 expressed by osteoblasts. Inhibiting Sema4D/Plexin-B1 signaling in the early-stage OA holds promise as a strategy for new therapeutic approaches to osteoarthritis.
\end{abstract}

\title{
Key words
}

Temporomandibular joint; Osteoarthritis; Semaphorin 4D; Plexin-B1; Osteoblast. 


\section{Introduction}

Temporomandibular joint (TMJ) osteoarthritis (OA) is the most severe pattern of temporomandibular joint disorder (TMD), with characteristics of degeneration of condylar cartilage and abnormal subchondral bone remodeling ${ }^{[1]}$. Previously, osteoarthritis was regarded to originate from metabolic disorder and degradation of joint cartilage, so the studies were mainly focusing on cartilage degradation ${ }^{[2,3]}$. Recently, the important role of abnormal bone remodeling in the progressing of osteoarthritis gained much attention ${ }^{[4]}$. In physiological state, the subchondral bone played roles of absorbing and buffering stress and maintaining the shape of joint ${ }^{[5]}$. In the process of osteoarthritis, the abnormal remodeling of subchondral bone could accelerate the degradation of cartilage covering it ${ }^{[6,7]}$.

Studies on the late-stage OA reported that the main change of subchondral bone was osteosclerosis ${ }^{[8]}$, characterized by thickening of subchondral plate and increase of subchondral cancellous bone mineral density and bone mass ${ }^{[9,10]}$. However, it is hard to reverse late-stage joint degeneration, and joint replacement was the ultimate treatment method ${ }^{[11,12]}$. In the early-stage $\mathrm{OA}$ in knee joint of both patients and experimental animals, significant subchondral bone loss was observed, with decreased trabecular thickness, quantity and bone mineral density ${ }^{[13-15]}$. In our previous TMJ OA studies, subchondral bone loss was also supervised in early-stage TMJ OA [16-19]. The subchondral bone loss might be an important pathological feature of early-stage OA and a significant factor promoting OA progression. Therefore, to disclosure the pathological molecular mechanism of early-stage OA might help to delay the progression of OA by early intervention.

Bone remodeling involved two aspects, bone resorption and bone formation. The subchondral bone loss of early-stage OA was mainly attributed to increased activity of osteoclasts [16,20,21]. Drugs inhibiting osteoclasts activities have been improved effective in OA therapy ${ }^{[22]}$. On the other hand, the action of osteoblasts in the subchondral bone loss of early-stage OA has not got adequate attention ${ }^{[23]}$. Thus, besides the activity of osteoclasts, the changes of bone formation activity of osteoblasts might be another important link in the subchondral bone loss of early-stage $\mathrm{OA}$, and the mechanism of this process is still unclear.

Semaphorin 4D (Sema4D) and Plexin-B1, a pair of molecules, were recently reported to expressed in osteoclasts and osteoblasts, respectively, with a role of inhibiting the bone formation activity of osteoblasts ${ }^{[24]}$. In early-stage TMJ OA, we found that Sema4D and Plexin-B1 was up-regulated, but the role of Sema4D/Plexin-B1 in OA is still unknown. It was presumed that up-regulated Sema4D/Plexin-B1 in early-stage OA might participate in the subchondral bone loss by inhibiting the bone formation activity of osteoblasts, which might be an important mechanism of bone loss in early-stage OA. If it holds, blocking Sema4D/Plexin-B1 signaling in early-stage OA might interrupt the progression of TMJ OA, and providing a novel method of early intervention of OA.

\section{Materials and Methods}

\subsection{Animals and UAC procedure}

One hundred and eight C57BL/6J female mice (18-20g), 8 weeks of age, were provided by the Laboratory Animal Center of the Fourth Military Medical University (FMMU). 72 female mice 
used for 2, 4 and 8 weeks control and UAC group. In every group or timepoint, six left condyles were used for histology staining and six right condyles were used for Mico-CT analysis $(n=6)$. Another six left condyles were used for immunohistochemical staining $(n=6)$ and six right condyles were used for realtime-PCR and western blotting analysis (two samples in one duplicate, $\mathrm{n}=3$ ). Another thirty-six female mice used for TMJ injection at 2, 4 and 8 weeks and the sample details like UAC group $(n=6)$. All operations involving animals were conducted according to the U.K. Animals (Scientific Procedures) Act, 1986 and associated guidelines, complied with the ARRIVE guidelines and were approved by the Administration Committee of Experimental Animals in FMMU. The UAC procedure was performed as previously reported ${ }^{[16,19,25,26]}$. Briefly, under anesthesia with intraperitoneal pentobarbital sodium ( $40 \mathrm{mg} / \mathrm{kg}$ body weight), UAC was created by bonding a pair of metal tubes onto the left side maxillary and mandibular incisors.

\subsection{Histology, immunohistochemical (IHC) and immunofluorescent (IF) staining}

The TMJ condyle was fixed in 4\% paraformaldehyde, decalcified with 4\% Ethylene diamine tetraacetic acid (EDTA) solution for 4 weeks at room temperature, dehydrated in ethanol and embedded in paraffin wax. Consecutive slices (thickness, $4 \mu \mathrm{m}$ ) were prepared in the sagittal plane for Hematoxylin \& Eosin (H\&E), Safranin O and tartrate-resistant acid phosphatase (TRAP) staining, and also for IHC and IF staining. The most central sagittal sections of each joint were selected. The primary antibodies for IHC and IF staining were anti-Semaphorin 4D (ab231961, 1:100 dilution), anti-Plexin-B1 (sc-28372, 1:100 dilution), anti-Cathepsin K (sc-48353, 1:200 dilution) and anti-Osteocalcin (ab93876, 1:100 dilution). In the H\&E stained samples, the thickness of cartilage was evaluated as previously reported ${ }^{[16]}$. TRAP staining was performed following the manufacturer's instructions (Sigma 387-A, St Louis, USA). Quantification of integral optical density (IOD) of Safranin O staining, the number of TRAP positive cells and percentage of Sema4D positive cells were performed as previously reported ${ }^{[25,27]}$. For assessing IOD of Plexin-B1, Cathepsin K, OCN and double positive area in the subchondral bone, one square $(0.3 \mathrm{~mm} \times 0.3 \mathrm{~mm})$ located beneath the osteochondral interface of the central part of the condyle was chosen, and all the parts besides blood vessels were included for analysis. The data were averaged from 3 slides of each animal.

\subsection{Micro-CT}

The dissected TMJ condyles were fixed overnight in $4 \%$ paraformaldehyde and analyzed by high-resolution micro-CT (Y. Cheetah; YXLON, Hamburg, Germany). Scanning was performed at $90 \mathrm{kV}$ and $100 \mu \mathrm{A}$ with a resolution of $4.6 \mu \mathrm{m} / \mathrm{pixel}$. The sagittal images of the condyle were used to assess the thickness of cartilage. In the 3-dimensional images, 2 cubic regions of interest $(0.3 \times$ $0.3 \times 0.3 \mathrm{~mm})$ at the middle points of the center and posterior condyle were selected to examine subchondral bone as previously described ${ }^{[28]}$. The ratios of bone volume (BV/TV), trabecular thickness (Tb.Th), trabecular separation (Tb.Sp), and trabecular number (Tb.N) were measured.

\subsection{Realtime-PCR}

After the animals were euthanized under anesthesia, the cartilage and subchondral bone of the TMJ condyles in each subgroup were carefully dissected out under a stereomicroscope and preserved at $-80^{\circ} \mathrm{C}$ for messenger Ribonucleic Acid (mRNA) preparation, respectively.

The total mRNA in the cartilage and subchondral bone was extracted by Tripure buffer (Roche, 
11667165001, Germany), respectively. Reverse transcription of the mRNA to template complementary deoxyribonucleic acid (cDNA) was completed using a TIANScript RT Kit (Tiangen, Beijing, China). Genes were analyzed using the Applied Biosystems 7500 Realtime-PCR machine (Applied Biosystems, Foster City, CA, USA). Each experiment was performed three times and the mean values were derived. The amount of target cDNA, relative to Gapdh, was calculated using the formula $2^{-\Delta \Lambda} \mathrm{Ct}$, where $\mathrm{Ct}$ means Cycle threshold. The results were calculated as the relative quantification of the target gene compared to the age-matched control group, which was set at 1 . All primers used were shown in Table 1.

\subsection{Western blotting}

Total lysates of tissues were obtained by Tripure buffer (Roche, 11667165001, Germany) with a protease inhibitor cocktail (Sigma-Aldrich, St Louis, USA). For western blotting, 20 ug total proteins were separated by SDS PAGE and transferred to a polyvinylidene fluoride (PVDF) membrane (Immobilon-P; Millipore, MA, USA), after incubated with 5\% BSA, which was probed with the primary antibodies for anti-Semaphorin 4D (ab39710, 1:1000 dilution), anti-Plexin-B1 (sc-28372, 1:200 dilution) and anti-GAPDH (ab8245, 1:2000 dilution). After incubation with the secondary antibodies, proteins were visualized by using an enhanced chemiluminescence (ECL) system (Biorad, USA).

\subsection{Injections of drugs}

The injection of BMA-12 (MBL) was carried out as follows ${ }^{[25,26]}$. After the induction of deep anesthesia, the mouse was laid sidelong. A specially made microinjector was inserted at the point just below the zygomatic arch until the outer surface of the mandibular ramus was reached. The orientation of the needle head was adjusted to make the needle pass along the bone wall and finally reach the TMJ region. BMA-12 were injected into the TMJ region through the needle. In the control group, physiological saline was injected.

\subsection{MC3T3-E1 cell culture}

The murine osteoblast cell line (MC3T3-E1) was obtained from American Type Culture Collection (ATCC). Cells were cultured in $\alpha$-MEM supplemented with $10 \%$ FBS, $100 \mathrm{U} / \mathrm{ml}$ streptomycin and $100 \mathrm{U} / \mathrm{ml}$ penicillin and $2 \mathrm{mM} \mathrm{L}$-glutamine at $37{ }^{\circ} \mathrm{C}$ in a humidified atmosphere of $95 \%$ air and $5 \% \mathrm{CO}_{2}$. For osteogenic differentiation, the medium was replaced with osteogenic differentiation medium ( $50 \mu \mathrm{M}$ ascorbic acid, $10 \mathrm{nM}$ dexamethasone and $10 \mathrm{mM}$

$\beta$-glycerophosphate). After 7 days culturing, MC3T3-E1 cells were transfected with control vector or the Plxnb1 vector (GV287, Genechem, Shanghai, China). All experiments were repeated in at least three independent tests. Neutralizing antibodies against Sema4D (BMA-12), recombinant Sema4D (Fc-sema4D) (20 $\mu$ g, unless otherwise indicated), or BMA-12 and Fc-sema4D simultaneously, were added every 3 days from the eighth day of culturing. Then after 21 days, cells were collected and the total mRNA was extracted for realtime-PCR analysis of osteoblast-related genes.

\subsection{Transwell migrating assay}

Cell migration was assessed using a transwell chamber assay as previously reported ${ }^{[28]}$. A $96-$-well microchemotaxis chamber with $8-\mu \mathrm{m}$ pores was used. Two thousand cells in a $20-\mu \mathrm{L}$ 
volume were placed in the upper chamber. BMA-12, Fc-sema4D, or BMA-12 and Fc-sema4D (20 $\mu \mathrm{M})$ simultaneously, were added to the lower chamber. After 24-h incubation, the cells that migrated to the lower surface of the membrane were stained with 4',6-diamidino-2-phenylindole (DAPI) and observed under an Olympus microscope (Tokyo, Japan) at 200×. Each well was divided into 9 parts by imaginarily drawing a "\#” under the microscope. Images were obtained from the center of each part for cell counting, and the means of 5 slides were used for analysis.

\subsection{Statistical analysis}

The data were expressed as means and standard deviations. The statistical significance of the differences between 2 groups was analyzed by Student's $t$-test, and that among 3 or more groups was evaluated by one-way analysis of variance (ANOVA) followed by the Tukey Post-Hoc test for pair-wise comparison. All statistical analyses were performed with SPSS software (version 22.0 for Windows; SPSS, Chicago, IL). The level of significance was determined by $P<0.05$ for all statistical tests.

\section{Results}

\subsection{The degeneration of cartilage and subchondral bone loss in TMJ condyle with early-stage OA}

The TMJ OA in mice was induced in our previously reported unilateral anterior crossbite (UAC) model ${ }^{[16,19,25,26]}$. Hematoxylin-eosin (H\&E) staining showed a significant thinner condylar cartilage in $2 \mathrm{w}, 4 \mathrm{w}$ and $8 \mathrm{w}$ UAC groups (Fig 1A), with $P<0.05$ (Fig 1B). Cartilage matrix loss was also observed in UAC group by Safranin O staining (Fig 1C), with significant decreased positive staining area in UAC group (Fig 1D). The subchondral bone loss was significant in $2 \mathrm{w}$, $4 \mathrm{w}$ and $8 \mathrm{w}$ UAC groups (Fig 2A), and Micro-CT morphological analysis of subchondral bone showed deceased BV/TV (bone volume per trabecular volume) and Tb.Th (trabecular thickness), and increased Tb.Sp (trabecular separation), also indicating bone loss (Fig 2B). The Tb.N has no change (Fig 2B).

\subsection{The changes of Sema4D and Plexin-B1 in condyle with early-stage TMJ OA}

The immunohistochemical staining showed Sema4D was expressed at both articular cartilage and subchondral bone in control group. UAC increased Sema4D positive cells in both articular cartilage and subchondral bone in UAC group, compared with control group (Fig 3A and B). Increased Plexin-B1 IHC-positive staining was observed in the subchondral bone in UAC group, and little Plexin-B1 positive staining was observed in the subchondral bone in control group and in articular cartilage in both control and UAC groups (Fig 3C and D). Realtime-PCR results showed that the gene expression of Sema4D in the articular cartilage and subchondral bone increased in $2 \mathrm{w}, 4 \mathrm{w}$ and $8 \mathrm{w}$ UAC groups compared with control group (Fig 3E). The Plexin-B1 expressed in subchondral bone, but not in articular cartilage, and increased in $2 \mathrm{w}, 4 \mathrm{w}$ and $8 \mathrm{w}$ UAC groups compared with control group (Fig 3E). Western-blotting results showed that the protein expression of Sema4D in the articular cartilage and subchondral bone increased in UAC groups, compared with control group. The protein expression of Plexin-B1 expression in subchondral bone was also significantly increased in UAC groups, compared with control group (Fig 3F). However, there was little detection of Plexin-B1 in articular cartilage (Fig 3F). To further 
locate the expressed position of Sema4D and Plexin-B1, double-labeling immunofluorescence showed that Sema4D expression was mainly increased in Cathepsin K-positive osteoclasts in subchondral bone at $8 \mathrm{w}$ group (Fig 4A and B). Moreover, Plexin-B1 expression was mainly increased in OCN-positive cells in subchondral bone in the 8w UAC group (Fig 4C and D). This data demonstrated that obviously change of Sema4D/Plexin-B1 signaling under osteoarthritis.

\subsection{The changes of osteoblasts and osteoclasts in condyle with early-stage TMJ OA}

Next, we detected osteoclasts number in subchondral bone by TRAP staining. The number of osteoclasts indicated by TRAP-positive expression was increased in $2 \mathrm{w}, 4 \mathrm{w}$ and $8 \mathrm{w}$ UAC groups, compared with control group (Fig 5A and B). The gene expression of two osteoblast differentiation-associated factor, Runx 2 and OSX, in subchondral bone of UAC group has no significant changes compared with that of control group at $2 \mathrm{w}$ time points, but was increased at $4 \mathrm{w}$ and $8 \mathrm{w}$ time points (Fig 5C). Gene expression of OCN and ALP, which was associated with the bone-producing function of osteoblasts, displayed a similar changing tendency in subchondral bone of UAC group, which was increased at $4 \mathrm{w}$ and $8 \mathrm{w}$ time points (Fig 5C). This data revealed that UAC could upregulate the number of osteoclasts and the activity of osteoblasts accompanied with phenotype of subchondral bone loss.

\subsection{Inhibiting Sema4D reversed early degeneration of TMJ OA}

To further identify the function of Sema4D signaling in inhibiting bone formation activity of osteoblasts, weekly TMJ local area injection of BMA-12, a neutralizing antibody of Sema4D, was performed. BMA-12 obviously decreased the positive expression staining of Sema4D in the condyle in 2, 4 and 8w group (Fig S1). After 8 weeks inhibition of Sema4D, it reversed the decrease of condylar cartilage thickness (Fig 6A and B). The amount of cartilage matrix in the BMA-12 injection group was increased than that in UAC group, indicated by Safranin O staining (Fig 6C and D). Micro-CT analysis of subchondral bone showed that local injection of BMA-12 reversed the condylar subchondral bone loss (Fig 6E), indicated by the increase of BV/TV, Tb.Th, and decreased Tb.Sp in the 8w BMA-12 group (Fig 6F).

\subsection{The changes of osteoblasts and osteoclasts in condylar subchondral bone after local injection of Sema4D inhibitor}

We next detected the changes of osteoblasts and osteoclasts after the inhibition of Sema4D. After 8 weeks local injection of BMA-12, the osteoclasts number indicated by TRAP staining in condylar subchondral bone in BMA-12 injection group was significantly decreased than that in UAC group (Fig 7A and B). However, the gene expression of Runx2, OSX, OCN and ALP in subchondral bone of BMA-12 group has no significant difference with that of UAC group, which was still up-regulated compared with control group (Fig 7C). This assay confirmed that inhibition of Sema4D could reduce the number of osteoclasts, but not influence the number and activity of osteoblasts.

\subsection{Migrating ability and gene expression of osteoblasts was changed by Sema4D inhibitor in vitro}

The transwell migrating assay was carried out to reveal the specific impact of Sema4D to osteoblasts in vitro. Results showed that Fc-Sema4D and BMA-12, added either separately or 
simultaneously, induced no significant changes of spontaneous migration of osteoblasts. While after the osteoblasts were transfected with Plxnb1 (the gene of Plexin-B1), Fc-Sema4D induced significant increased migration of osteoblasts. When BMA-12 added simultaneously with Fc-Sema4D to the transwell assay, the increased migration of Plxnbl-transefected osteoblasts induced by Fc-Sema4D was reversed (Fig 8A and B). The gene expression changes of both Plxnb1-untransfected and -transfected osteoblasts induced by BMA-12 and Fc-Sema4D were detected by realtime-PCR. The gene expression of Runx2, OSX, OCN and ALP of untransfected osteoblasts was not influenced by Fc-sema4D and BMA-12, either separately or simultaneously. Fc-Sema4D induced a decrease of OCN and ALP gene expression of Plxnb1-transfected osteoblasts, which was reversed by BMA-12 addition (Fig 8C).

\section{Discussion}

$\mathrm{OA}$ is the most common joint disease, which is characterized by degeneration of articular cartilage and abnormal changes of subchondral bone. Subchondral bone of normal structure guarantees the integrity of the overlying articular cartilage ${ }^{[29]}$. The articular cartilage degeneration is always accompanied by microstructural changes of subchondral bone ${ }^{[30]}$. The microstructural changes of subchondral bone could influence its mechanical behavior, which influence the tolerance of articular cartilage to mechanical stress by the crosstalk between articular cartilage and subchondral bone ${ }^{[31]}$. If the abnormal subchondral bone changes were not reversed, the overlying articular cartilage degeneration is not likely to be repaired. In other words, a treatment of osteoarthritis could not success without managing subchondral bone.

It is commonly accepted that subchondral bone plays an important role in the development of $\mathrm{OA}^{[32-34]}$. In the early-stage OA, the subchondral bone changes mainly display as subchondral bone loss, which was attributed as one of the key reasons of the development of OA. In consistency with our previous study ${ }^{[16,19,28]}$, the present study reported a significant subchondral bone loss in the UAC-induced early-stage TMJ OA. The subchondral bone goes through dynamic remodeling, during which bone resorption and new bone formation was in balance in physiological condition ${ }^{[35-37]}$. The subchondral bone loss of early-stage OA is mainly attributed to the elevated osteoclasts number and enhanced osteoclasts activity ${ }^{[28,38]}$.

However, the balance of bone resorption and reformation is depended on the activity of not only osteoclasts, but also osteoblasts ${ }^{[39-41]}$. The balance of subchondral bone is depended on both osteoclasts and osteoblasts. But the activity of osteoblasts in early-stage OA has not drawn enough attention. The present study found that in the UAC-induced early-stage TMJ OA, there was an increase of the genes expression of Runx2, OSX, OCN and ALP in the subchondral bone, implying that osteoblasts differentiation was enhanced. However, a success bone forming activity of osteoblasts involves many issues.

The bone-forming process is mainly accomplished by osteoblasts. The differentiation, migration and the capability of expressing bone-formation-related molecules are all important issues deciding the bone-forming ability of osteoblasts ${ }^{[24]}$. It has been reported that osteoblasts from OA tissue fail to mineralize normally, although presenting high expression of markers of osteoblast/osteocyte-like cells ${ }^{[42]}$. The impaired bone-forming activity of osteoblasts in early-stage OA should be attributed to other reasons than their differentiation. It has been indicated 
that increased motility of osteoblasts could repel the bone-forming activity of osteoblasts in a model of postmenopausal osteoporosis ${ }^{[24]}$.

Sema4D, a transmembrane protein, could be proteolytically cleaved into a soluble form once the cell was activated, and was reported to expressed by osteoclasts ${ }^{[43,44]}$. Plexin-B1 is the specific receptor of Sema4D, which was induced to be expressed by osteoblasts during differentiation. Sema4D could binding to the Plexin-B1 and inhibits osteoblastic bone formation by stimulating osteoblasts motility in an osteoporosis model ${ }^{[24]}$. In the present study, we found that Sema4D expression was up-regulated in subchondral bone of early-stage TMJ OA, especially in the Cathepsin K-positive osteoclasts. Moreover, we found for the first time that Sema4D could also be expressed in articular cartilage, and the expression was elevated in early-stage TMJ OA. Plexin-B1 was at a very low expression level in normal TMJ. But in subchondral bone of early-stage OA, particularly in OCN-positive osteoblasts, the expression of Plexin-B1 was positive. Local injection of BMA-12 inhibited the subchondral bone loss of early-stage OA, accompanied by improvement of cartilage thickness and recovery of cartilage matrix. The increased expression of Runx2, OSX, OCN and ALP in subchondral bone was not changed by BMA-12 local injection. Therefore, the most rational explanation was that the injected BMA-12 inhibited the enhanced osteoblasts migration stimulated by Sema4D/Plexin-B1 signaling, and thus improve the bone-forming activity of osteoblasts and inhibited the subchondral bone loss. And the ameliorated subchondral bone structure was beneficial for the repairing of the overlying cartilage [29, 30].

For next step by transwell migrating assay, data also showed that Sema4D could enhance the migration of Plexin-B1 positive osteoblasts, but not in Plexin-B1 negative osteoblasts. And BMA-12, a Sema-4D inhibitor, reversed the increased migration of osteoblasts. It is possible that the increased expression of soluble Sema4D by osteoclasts in subchondral bone and in articular cartilage enhanced the motility of osteoblasts by binding to Plexin-B1 expressed by osteoblasts, and inhibits osteoblastic bone formation. This could be an explanation for that although expression of Runx2, OSX, OCN and ALP in the subchondral bone was increased, the subchondral bone loss was still significant in early-stage OA.

In our in vitro study, Sema4D could reduce the expression of OCN and ALP in Plexin-B1 positive osteoblast, which was similar to previous report ${ }^{[45]}$. However, in vivo study of UAC-induced early-stage OA, the expression of Runx2, OSX, OCN and ALP was increased. Many factors could stimulate the expression of these molecules of osteoblasts in vivo, including TGF- $\beta$, HGF and IGF, which were frequently reported to increase in early-stage OA ${ }^{[38,46-49]}$. Therefore, the function of Sema4D/Plexin-B1 in the differentiation of osteoblasts in early-stage OA might be offset by these factors in vivo.

In conclusion, we demonstrated that, in early-stage TMJ OA, increased production of Sema4D in condylar cartilage and subchondral bone and elevated Plexin-B1 expression in osteoblasts are responsible, at least in part, for the inhibited bone-forming activity of osteoblasts. The present study provided a novel insight of subchondral bone loss of early-stage OA. Inhibiting Sema4D/Plexin-B1 signaling in the early-stage OA holds promise as a strategy for new therapeutic approaches to OA.

\section{Declaration of Competing Interest}


The authors declare no potential conflicts of interest with respect to the authorship and/or publication of this article.

\section{Acknowledgments}

We gratefully acknowledge Shujing Cai for assistance in animal experiments and histology procedures. This work was supported by the National Natural Science Foundation of China (grants 82001071, 81970953 and 82001072), and the Basic Natural Science Foundation of Shanxi Province (2020JQ-453).

Author contribution: HY, JF, and LL designed the research. ZZ, TY, MZ, JZ, FH and QL collected the data. LL, ZZ, MZ and JZ contributed to the statistical analysis. LL, HY, JF and SY contributed to the interpretation of the data. All authors contributed in revising the manuscript critically and final approval of the version to be submitted.

\section{Reference}

1. Zhang S, Teo KYW, Chuah SJ, Lai RC, Lim SK, Toh WS. MSC exosomes alleviate temporomandibular joint osteoarthritis by attenuating inflammation and restoring matrix homeostasis. Biomaterials. 2019 Apr;200:35-47. doi: 10.1016/j.biomaterials.2019.02.006.

2. Mobasheri A, Rayman MP, Gualillo O, Sellam J, van der Kraan P, Fearon U. The role of metabolism in the pathogenesis of osteoarthritis. Nat Rev Rheumatol. 2017 May;13(5):302-311. doi: 10.1038/nrrheum.2017.50.

3. Valdes AM. Metabolic syndrome and osteoarthritis pain: common molecular mechanisms and potential therapeutic implications. Osteoarthritis Cartilage. 2020 Jan;28(1):7-9. doi: 10.1016/j.joca.2019.06.015.

4. Felson DT, Neogi T. Osteoarthritis: is it a disease of cartilage or of bone? Arthritis Rheum. 2004 Feb;50(2):341-4. doi: 10.1002/art.20051.

5. Mansell JP, Collins C, Bailey AJ. Bone, not cartilage, should be the major focus in osteoarthritis. Nat Clin Pract Rheumatol. 2007 Jun;3(6):306-7. doi: 10.1038/ncprheum0505.

6. Glyn-Jones S, Palmer AJ, Agricola R, Price AJ, Vincent TL, Weinans H, Carr AJ. Osteoarthritis. Lancet. 2015 Jul 25;386(9991):376-87. doi: 10.1016/S0140-6736(14)60802-3.

7. Bailey AJ, Mansell JP. Do subchondral bone changes exacerbate or precede articular cartilage destruction in osteoarthritis of the elderly? Gerontology. 1997;43(5):296-304. doi: $10.1159 / 000213866$.

8. Burr DB, Gallant MA. Bone remodelling in osteoarthritis. Nat Rev Rheumatol. 2012 Nov;8(11):665-73. doi: 10.1038/nrrheum.2012.130.

9. Schulze-Tanzil G. Intraarticular Ligament Degeneration Is Interrelated with Cartilage and Bone Destruction in Osteoarthritis. Cells. 2019 Aug 27;8(9):990. doi: 10.3390/cells8090990.

10. Goldring SR, Goldring MB. Changes in the osteochondral unit during osteoarthritis: structure, function and cartilage-bone crosstalk. Nat Rev Rheumatol. 2016 Nov;12(11):632-644. doi: 10.1038/nrrheum.2016.148.

11. Bettica P, Cline G, Hart DJ, Meyer J, Spector TD. Evidence for increased bone resorption in patients with progressive knee osteoarthritis: longitudinal results from the Chingford study. Arthritis Rheum. 2002 Dec;46(12):3178-84. doi: 10.1002/art.10630.

12. Dedrick DK, Goldstein SA, Brandt KD, O'Connor BL, Goulet RW, Albrecht M. A longitudinal study of subchondral plate and trabecular bone in cruciate-deficient dogs with osteoarthritis followed up for 54 months. Arthritis Rheum. 1993 Oct;36(10):1460-7. doi: 10.1002/art.1780361019.

13. Florea C, Malo MK, Rautiainen J, Mäkelä JT, Fick JM, Nieminen MT, Jurvelin JS, Davidescu A, Korhonen RK. Alterations in subchondral bone plate, trabecular bone and articular cartilage properties of rabbit femoral condyles at 4 weeks after anterior cruciate ligament transection. Osteoarthritis Cartilage. 2015 Mar;23(3):414-22. doi: 10.1016/j.joca.2014.11.023. 
14. Intema F, Hazewinkel HA, Gouwens D, Bijlsma JW, Weinans H, Lafeber FP, Mastbergen SC. In early OA, thinning of the subchondral plate is directly related to cartilage damage: results from a canine ACLT-meniscectomy model. Osteoarthritis Cartilage. 2010 May;18(5):691-8. doi: 10.1016/j.joca.2010.01.004.

15. Batiste DL, Kirkley A, Laverty S, Thain LM, Spouge AR, Holdsworth DW. Ex vivo characterization of articular cartilage and bone lesions in a rabbit ACL transection model of osteoarthritis using MRI and micro-CT. Osteoarthritis Cartilage. 2004 Dec;12(12):986-96. doi: 10.1016/j.joca.2004.08.010.

16. Lu L, Huang J, Zhang X, Zhang J, Zhang M, Jing L, Yu S, Wang M. Changes of temporomandibular joint and semaphorin 4D/Plexin-B1 expression in a mouse model of incisor malocclusion. J Oral Facial Pain Headache. 2014 Winter;28(1):68-79. doi: 10.11607/jop.1082.

17. Jiao K, Niu LN, Wang MQ, Dai J, Yu SB, Liu XD, Wang J. Subchondral bone loss following orthodontically induced cartilage degradation in the mandibular condyles of rats. Bone. 2011 Feb;48(2):362-71. doi: 10.1016/j.bone.2010.09.010.

18. Zhang J, Jiao K, Zhang M, Zhou T, Liu XD, Yu SB, Lu L, Jing L, Yang T, Zhang Y, Chen D, Wang MQ. Occlusal effects on longitudinal bone alterations of the temporomandibular joint. J Dent Res. 2013 Mar;92(3):253-9. doi: 10.1177/0022034512473482.

19. Liu YD, Liao LF, Zhang HY, Lu L, Jiao K, Zhang M, Zhang J, He JJ, Wu YP, Chen D, Wang MQ. Reducing dietary loading decreases mouse temporomandibular joint degradation induced by anterior crossbite prosthesis. Osteoarthritis Cartilage. 2014 Feb;22(2):302-12. doi: 10.1016/j.joca.2013.11.014.

20. Yu DG, Ding HF, Mao YQ, Liu M, Yu B, Zhao X, Wang XQ, Li Y, Liu GW, Nie SB, Liu S, Zhu ZA. Strontium ranelate reduces cartilage degeneration and subchondral bone remodeling in rat osteoarthritis model. Acta Pharmacol Sin. 2013 Mar;34(3):393-402. doi: 10.1038/aps.2012.167.

21. Hayami T, Pickarski M, Zhuo Y, Wesolowski GA, Rodan GA, Duong LT. Characterization of articular cartilage and subchondral bone changes in the rat anterior cruciate ligament transection and meniscectomized models of osteoarthritis. Bone. 2006 Feb;38(2):234-43. doi: 10.1016/j.bone.2005.08.007.

22. Lampropoulou-Adamidou K, Dontas I, Stathopoulos IP, Khaldi L, Lelovas P, Vlamis J, Triantafillopoulos IK, Papaioannou NA. Chondroprotective effect of high-dose zoledronic acid: An experimental study in a rabbit model of osteoarthritis. J Orthop Res. 2014 Dec;32(12):1646-51. doi: 10.1002/jor.22712.

23. Pelletier JP, Boileau C, Brunet J, Boily M, Lajeunesse D, Reboul P, Laufer S, Martel-Pelletier $\mathrm{J}$. The inhibition of subchondral bone resorption in the early phase of experimental dog osteoarthritis by licofelone is associated with a reduction in the synthesis of MMP-13 and cathepsin K. Bone. 2004 Mar;34(3):527-38. doi: 10.1016/j.bone.2003.11.021.

24. Negishi-Koga T, Shinohara M, Komatsu N, Bito H, Kodama T, Friedel RH, Takayanagi H. Suppression of bone formation by osteoclastic expression of semaphorin 4D. Nat Med. 2011 Oct 23;17(11):1473-80. doi: 10.1038/nm.2489.

25. Zhang M, Yang H, Wan X, Lu L, Zhang J, Zhang H, Ye T, Liu Q, Xie M, Liu X, Yu S, Guo S, Chang W, Wang M. Prevention of Injury-Induced Osteoarthritis in Rodent Temporomandibular Joint by Targeting Chondrocyte CaSR. J Bone Miner Res. 2019 Apr;34(4):726-738. doi: 10.1002/jbmr.3643.

26. Yang H, Wen Y, Zhang M, Liu Q, Zhang H, Zhang J, Lu L, Ye T, Bai X, Xiao G, Wang M. MTORC1 coordinates the autophagy and apoptosis signaling in articular chondrocytes in osteoarthritic temporomandibular joint. Autophagy. $2020 \mathrm{Feb} ; 16(2): 271-288$. doi: 10.1080/15548627.2019.1606647.

27. Liu Y, Liao L, Zhang H, Lu L, Jiao K, Zhang M, Zhang J, He J, Wu Y, Chen D, Wang M. Reducing dietary loading decreases mouse temporomandibular joint degradation induced by anterior crossbite prosthesis. Osteoarthritis and Cartilage. 2014;22(2): 302-312. doi:10.1016/j.joca.2013.11.014.

28. Lu L, Zhang X, Zhang M, Zhang H, Liao L, Yang T, Zhang J, Xian L, Chen D, Wang M. RANTES and SDF-1 Are Keys in Cell-based Therapy of TMJ Osteoarthritis. J Dent Res. 2015 Nov;94(11):1601-9. doi: 10.1177/0022034515604621.

29. Cui Z, Crane J, Xie H, Jin X, Zhen G, Li C, Xie L, Wang L, Bian Q, Qiu T, Wan M, Xie M, 
Ding S, Yu B, Cao X. Halofuginone attenuates osteoarthritis by inhibition of TGF- $\beta$ activity and H-type vessel formation in subchondral bone. Ann Rheum Dis. 2016 Sep;75(9):1714-21. doi: 10.1136/annrheumdis-2015-207923.

30. Gomoll AH, Madry H, Knutsen G, van Dijk N, Seil R, Brittberg M, Kon E. The subchondral bone in articular cartilage repair: current problems in the surgical management. Knee Surg Sports Traumatol Arthrosc. 2010 Apr;18(4):434-47. doi: 10.1007/s00167-010-1072-x.

31. Buckland-Wright C. Subchondral bone changes in hand and knee osteoarthritis detected by radiography. Osteoarthritis Cartilage. 2004;12 Suppl A:S10-9. doi: 10.1016/j.joca.2003.09.007.

32. Muraoka T, Hagino H, Okano T, Enokida M, Teshima R. Role of subchondral bone in osteoarthritis development: a comparative study of two strains of guinea pigs with and without spontaneously occurring osteoarthritis. Arthritis Rheum. 2007 Oct;56(10):3366-74. doi: 10.1002/art.22921.

33. Henrotin Y, Pesesse L, Sanchez C. Subchondral bone in osteoarthritis physiopathology: state-of-the art and perspectives. Biomed Mater Eng. 2009;19(4-5):311-6. doi: 10.3233/BME-2009-0596.

34. Botter SM, van Osch GJ, Waarsing JH, van der Linden JC, Verhaar JA, Pols HA, van Leeuwen JP, Weinans H. Cartilage damage pattern in relation to subchondral plate thickness in a collagenase-induced model of osteoarthritis. Osteoarthritis Cartilage. 2008 Apr;16(4):506-14. doi: 10.1016/j.joca.2007.08.005.

35. Martin TJ, Sims NA. Osteoclast-derived activity in the coupling of bone formation to resorption. Trends Mol Med. 2005 Feb;11(2):76-81. doi: 10.1016/j.molmed.2004.12.004.

36. Xian L, Wu X, Pang L, Lou M, Rosen CJ, Qiu T, Crane J, Frassica F, Zhang L, Rodriguez JP, Xiaofeng Jia, Shoshana Yakar, Shouhong Xuan, Argiris Efstratiadis, Mei Wan, Xu Cao. Matrix IGF-1 maintains bone mass by activation of mTOR in mesenchymal stem cells. Nat Med. 2012 Jul;18(7):1095-101. doi: 10.1038/nm.2793.

37. Zaidi M. Skeletal remodeling in health and disease. Nat Med. 2007 Jul;13(7):791-801. doi: $10.1038 / \mathrm{nm} 1593$.

38. Yang T, Zhang J, Cao Y, Zhang M, Jing L, Jiao K, Yu S, Chang W, Chen D, Wang M. Wnt5a/Ror2 mediates temporomandibular joint subchondral bone remodeling. J Dent Res. 2015 Jun;94(6):803-12. doi: 10.1177/0022034515576051.

39. Abe E, Marians RC, Yu W, Wu XB, Ando T, Li Y, Iqbal J, Eldeiry L, Rajendren G, Blair HC, Davies TF, Zaidi M. TSH is a negative regulator of skeletal remodeling. Cell. 2003 Oct 17;115(2):151-62. doi: 10.1016/s0092-8674(03)00771-2.

40. Mundy GR, Elefteriou F. Boning up on ephrin signaling. Cell. 2006 Aug 11;126(3):441-3. doi: 10.1016/j.cell.2006.07.015.

41. Zhen G, Wen C, Jia X, Li Y, Crane JL, Mears SC, Askin FB, Frassica FJ, Chang W, Yao J, Carrino JA, Cosgarea A, Artemov D, Chen Q, Zhao Z, Zhou X, Riley L, Sponseller P, Wan M, Lu WW, Cao X. Inhibition of TGF- $\beta$ signaling in mesenchymal stem cells of subchondral bone attenuates osteoarthritis. Nat Med. 2013 Jun;19(6):704-12. doi: 10.1038/nm.3143.

42. Couchourel D, Aubry I, Delalandre A, Lavigne M, Martel-Pelletier J, Pelletier JP, Lajeunesse D. Altered mineralization of human osteoarthritic osteoblasts is attributable to abnormal type I collagen production. Arthritis Rheum. 2009 May;60(5):1438-50. doi: 10.1002/art.24489.

43. Suzuki K, Kumanogoh A, Kikutani H. Semaphorins and their receptors in immune cell interactions. Nat Immunol. 2008 Jan;9(1):17-23. doi: 10.1038/ni1553.

44. Elhabazi A, Delaire S, Bensussan A, Boumsell L, Bismuth G. Biological activity of soluble CD100. I. The extracellular region of CD100 is released from the surface of T lymphocytes by regulated proteolysis. J Immunol. 2001 Apr 1;166(7):4341-7. doi: 10.4049/jimmunol.166.7.4341.

45. Deng X, Liang LN, Zhu D, Zheng LP, Yu JH, Meng XL, Zhao YN, Sun XX, Pan TW, Liu YQ. Wedelolactone inhibits osteoclastogenesis but enhances osteoblastogenesis through altering different semaphorins production. Int Immunopharmacol. 2018 Jul;60:41-49. doi: 10.1016/j.intimp.2018.04.037.

46. Wang D, Yang H, Zhang M, Zhang H, Lu L, Zhang J, Wan X, Wang M. Insulin-like growth factor-1 engaged in the mandibular condylar cartilage degeneration induced by experimental unilateral anterior crossbite. Arch Oral Biol. 2019 Feb;98:17-25. doi:

10.1016/j.archoralbio.2018.11.002. 
47. Massicotte F, Fernandes JC, Martel-Pelletier J, Pelletier JP, Lajeunesse D. Modulation of insulin-like growth factor 1 levels in human osteoarthritic subchondral bone osteoblasts. Bone. 2006 Mar;38(3):333-41. doi: 10.1016/j.bone.2005.09.007.

48. Chan TF, Couchourel D, Abed E, Delalandre A, Duval N, Lajeunesse D. Elevated Dickkopf-2 levels contribute to the abnormal phenotype of human osteoarthritic osteoblasts. J Bone Miner Res. 2011 Jul;26(7):1399-410. doi: 10.1002/jbmr.358.

49. Guévremont M, Martel-Pelletier J, Massicotte F, Tardif G, Pelletier JP, Ranger P, Lajeunesse D, Reboul P. Human adult chondrocytes express hepatocyte growth factor (HGF) isoforms but not $\mathrm{HgF}$ : potential implication of osteoblasts on the presence of HGF in cartilage. J Bone Miner Res. 2003 Jun;18(6):1073-81. doi: 10.1359/jbmr.2003.18.6.1073. 


\section{Figure Legends}

Figure 1. UAC induced morphological changes and matrix loss of condylar cartilage. (A) The H\&E staining showed a significant thinner cartilage of TMJ condyle in $2 w, 4 w$ and $8 w$ UAC compared with control group. Scale bar $=100 \mu \mathrm{m}$. (B) The related quantitative data of cartilage thickness. (C) The Safranin O staining showed a significant loss of cartilage matrix of TMJ condyle in $2 \mathrm{w}, 4 \mathrm{w}$ and $8 \mathrm{w}$ UAC group. Scale bar $=100 \mu \mathrm{m}$. (D) The related quantitative data of IOD of Safranin O. Data are presented as means $\pm \mathrm{SD}, \mathrm{n}=6 . *: P<0.05$ in the indicated UAC group vs control group.

Figure 2. UAC lead to subchondral bone loss accompanied with changes of bone morphology analysis value. (A) Significant subchondral bone loss in TMJ condyle was observed by Micro-CT in $2 \mathrm{w}, 4 \mathrm{w}$ and $8 \mathrm{w}$ UAC group. (B) BV/TV and Tb.Th value was decreased in subchondral bone of TMJ condyle in $2 \mathrm{w}, 4 \mathrm{w}$ and $8 \mathrm{w}$ UAC group, and Tb.Sp value was increased in subchondral bone of TMJ condyle in $4 \mathrm{w}$ and $8 \mathrm{w}$ UAC group. Data are presented as means $\pm \mathrm{SD}, \mathrm{n}=6$. *: $P<0.05$ in the indicated UAC group vs control group.

Figure 3. Sema4D/Plexin-B1 had a significant variation under UAC stimulation. (A) Immunohistochemical staining of Sema4D showed Sema4D expression located at articular cartilage and subchondral bone in control groups. UAC increased the expression of Sema4D in both articular cartilage and subchondral bone of TMJ condyle in $2 \mathrm{w}, 4 \mathrm{w}$ and $8 \mathrm{w}$ timepoint. Scale bar $=50 \mu \mathrm{m}$. (B) Quantitative analysis of Sema4D in articular cartilage. (C) Immunohistochemical staining of Plexin-B1 showed increased Plexin-B1 expression in the subchondral bone of TMJ condyle in $4 \mathrm{w}$ and $8 \mathrm{w}$ UAC group. There was almost no expression in articular cartilage. Scale bar $=50 \mu \mathrm{m}$. (D) Quantitative analysis of Plexin-B1 in subchondral bone. (E) Realtime-PCR analysis of Sema4D mRNA expression in articular cartilage and subchondral bone of TMJ condyle; Plexin-B1 mRNA expression in articular cartilage and subchondral bone of TMJ condyle. (F) Western-blotting quantification of protein expression of Sema4D and Plexin-B1 in articular cartilage and subchondral bone of TMJ condyle. Data are presented as means $\pm \mathrm{SD}, \mathrm{n}=6$ for staining and $\mathrm{n}=3$ for realtime-PCR and Western-blotting. *: $P<0.05$ in the indicated UAC group vs control group.

Figure 4. Double-labeling immunofluorescent staining. (A) Expression of osteoclast marker, Cathepsin K, was increased in the subchondral bone in 8w UAC group. The increased Sema4D-positive area was widely double localized with Cathepsin K-positive area. White arrow, double staining of Sema4D and Cathepsin K. (B) The IOD of Cathepsin K-positive and Cathepsin $\mathrm{K}$ and Sema4D double-positive area was increased in 8w UAC group. (C) The Expression of osteoclast marker, OCN, was increased in the subchondral bone in $8 \mathrm{w}$ UAC group. The increased Plexin-B1-positive area was widely double localized with $\mathrm{OCN}$-positive area. White arrow, double staining of Plexin-B1 and OCN. (D) The IOD of OCN-positive and OCN and OCN and Plexin-B1 double-positive area was increased in $8 \mathrm{w}$ UAC group. Scale bar $=25 \mu \mathrm{m}$. Data are presented as means $\pm \mathrm{SD}, \mathrm{n}=6 . *: P<0.05$ in the indicated UAC group vs control group.

Figure 5. Biological change of osteoclasts under UAC stimulation. (A) TRAP-positive 
osteoclasts were increased in subchondral bone of TMJ condyle in 2w, $4 \mathrm{w}$ and 8w UAC group. Scale bar $=25 \mu \mathrm{m}$. (B) Quantitative data of TRAP-positive cells. (C) Realtime-PCR results showed Runx2, OSX, OCN and ALP mRNA expression was increased in the subchondral bone of TMJ condyle in $4 \mathrm{w}$ and $8 \mathrm{w}$ UAC group. Data are presented as means $\pm \mathrm{SD}, \mathrm{n}=6$ for staining and $\mathrm{n}=3$ for realtime-PCR. *: $P<0.05$ in the indicated UAC group vs control group.

Figure 6. Inhibition of Sema4D/Plexin-B1 could reverse the degeneration of condylar cartilage and subchondral bone. (A) H\&E staining of TMJ condyle after BMA-12, a neutralizing antibody of Sema4D, was weekly local-injected for 8 weeks. Scale bar $=100 \mu \mathrm{m}$. (B) The decreased thickness of cartilage induced by UAC was reversed by BMA-12 local injection. (C) The Safranin O staining of TMJ condyle after BMA-12 was weekly local injected for 8 weeks. Scale bar $=100 \mu \mathrm{m}$. (D) The decrease of cartilage matrix induced by UAC, indicating by deceased Safranin O positive staining, was reversed by BMA-12 local injection. (E) Subchondral bone loss in TMJ condyle in UAC group was reversed by BMA-12 local injection, observed by Micro-CT. (F) The decreased BV/TV and Tb.Th, and increased Tb.SP in TMJ condyle in UAC group was reversed by BMA-12 local injection. Data are presented as means $\pm \mathrm{SD}, \mathrm{n}=6 . *: P<0.05$ indicated the related groups.

Figure 7. BMA-12 reversed the osteoclasts activity. (A) The increased TRAP-positive osteoclasts in the subchondral bone of TMJ condyle in UAC group was reversed by BMA-12 local injection. Scale bar $=25 \mu \mathrm{m}$. (B) Quantitative data of TRAP-positive cells. (C) Realtime-PCR analysis showed that the increased Runx2, OSX, OCN and ALP mRNA expression in subchondral bone of TMJ condyle in UAC group was not influenced by BMA-12 local injection. Data are presented as means $\pm \mathrm{SD}, \mathrm{n}=6$ for staining and $\mathrm{n}=3$ for realtime-PCR. $*: P<0.05$ indicated the related groups.

\section{Figure 8. BMA-12 reversed the migrating ability of osteoblasts through Sema4D/Plexin-B1} signaling. (A) The observation of the transwell migrating assay showed that the number of migrated osteoblasts increased when they were Plexin-B1-positive and stimulated by Fc-sema4D, and this increase was inhibited by BMA-12. Scale bar $=20 \mu \mathrm{m}$. (B) The statistical results of number of migrated osteoblasts. The migrated cell number was not changed by Fc-Sema4D without Plxnbl transfection, but was increased by Fc-Sema4D after Plxnbl transfection, and this increase was inhibited by BMA-12. (C) Runx2, OSX, OCN and ALP mRNA expression of osteoblasts was not changed by Fc-Sema4D without Plxnbl transfection. OCN and ALP mRNA expression of osteoblasts was decreased after Plxnbl transfection, and this decrease was reversed by BMA-12. Data are presented as means $\pm \mathrm{SD}, \mathrm{n}=5$. *: $P<0.05$ indicated the related groups.

Figure S1. BMA-12 reversed the decreased of Sema4D expression. (A) Immunohistochemical staining of Sema4D in TMJ condyle after in $2 \mathrm{w}, 4 \mathrm{w}$ and $8 \mathrm{w}$ timepoint local injection of BMA-12. Scale bar $=50 \mu \mathrm{m}$. (B) Quantitative analysis of Sema4D in articular cartilage. Data are presented as means $\pm \mathrm{SD}, \mathrm{n}=6$. *: $P<0.05$ in the indicated UAC group vs control group. 
Table 1. Primer sequences for real-time-PCR

\begin{tabular}{lll}
\hline Gene & Forward & Reverse \\
\hline Runx2 & AAAGCCAGAGTGGACCCTTCCA & ATAGCGTGCTGCCATTCGAGGT \\
Osx & AGAGGTTCACTCGCTCTGACGA & TTGCTCAAGTGGTCGCTTCTG \\
Ocn & CCTGAGTCTGACAAAGCCTTCA & GCCGGAGTCTGTTCACTACCTT \\
Alp & CACAGATTCCCAAAGCACCT & GGGATGGAGGAGAGAAGGTC \\
Sema4d & GTTGATGATCCCGCGAGTTG & AGATCAGCCTGGCCTTTAGGAA \\
Plexinb1 & GGTCCACCTTGATTGCAGGTC & CACTGCCTGGAATCGCCTTTA \\
Gapdh & TGTGTCCGTCGTGGATCTGA & TTGCTGTTGAAGTCGCAGGAG \\
\hline
\end{tabular}

